\title{
Biomaterials: applications, manipulation, function and structure detection
}

\begin{abstract}
A.A. Mahmoud ${ }^{* 1}$, D. Atta ${ }^{1}$
${ }^{1}$ Spectroscopy department, Physics division, National Research Centre, 33 El Behooth St., Dokki, Giza, Egypt, Affiliation ID: 60014618.
\end{abstract}

The functionality of biomaterials is providing some advantages which enable it for targeted application these advantages are limited by the available techniques which is a topic of research to continue in the establishment of spectroscopic tools for investigation to follow up the changes in this critical and vital field of research.

Biomaterials and biological Molecules investigation needs certain care according to their sensitive role in one hand and low detection limits on the other hand. Many spectroscopic tools are already introduced including Raman technique, fluorescence as well as techniques based on single molecule detection.

The introduced techniques depending on conventional spectroscopic methods also it depends on imaging.

Biomaterials are achieved after blending and/or introduction of matrix include part or full metal oxides nano materials in order to facilitate the process of functionalization based on the desired applications.

Keywords: Biomaterial, biopolymers, biological molecules, FTIR, UV-Vis

Corresponding author: Prof. Abdelaziz Abd Elhalim Mahmoud, Basemabdo@yahoo.com 


\section{1- Classifications and general introduction of biomaterials}

Biopolymers are class of polymers which originated by living organisms; it could be also defined as the polymeric biomolecules. According to the nature of monomer constituting it, biopolymers could be classified into three main classes namely: The first class: Polynucleotides includes RNA as well as DNA, that are structures based on more or less 13 monomers; The second class is termed Polypeptides, formed from amino acids specially those of short structures; and finally, the third class: Polysaccharides, which are often of carbohydrate family [1].

In this state of art two classes of biopolymer will be under spot namely: Polypeptides such as the natural protein gelatin and Polysaccharides such as chitosan, cellulose and starch. Each one of the studied biopolymers will be discussed then their possible composites will be also discussed.

\section{1-1. Biopolymers}

Development of new active materials is a worldwide challenge, especially for those materials originate from natural biopolymers such as cellulose and its derivatives, chitosan as well as their derivatives, and starch. These materials are widely used in industrial applications including foodstuffs such as edible films, packaging and coating which require materials with biodegrade during the storage with controlled conditions in order to relieve the growing materials waste problem [2]. In this sense, chitosan and its derivatives could be considered among the most important materials according to its film-forming material and antimicrobial activity [3]. Chitosan could be described as a copolymer consists of pyranose ring of $\mathrm{N}$-acetyl-D-glucosamine (GlcNAc) and Nglucosamine (GluN) linked with a glycosidic linkage. It could be chemically derived from the deacetylation of chitin by a well-known chemical approach [4]. Chitosan is dedicated for many applications according to its biocompatibility, biodegradability and non-toxicity [5]. Among such applications food packaging, agricultural applications, also could be widely applied in biomedical and cosmetics domains while it is considered as promising too in drug delivery systems $[6,7]$. 
Chitosan films could be further improved in order to enhance its ability and applications as antimicrobial, antioxidant and/or mechanical properties, these improvements could be achieved with adding some active compounds. Practically, these modifications by addition may be carried out by chemical, physical and enzymatic approaches [8].

Another biopolymer which is subjected to extensive work according to its enormous application is cellulose. Cellulose is considered as the most abundant telluric material. It is renewable, inexpensive, it has very simple origin, which enable scientist to extract it from cell wall of plant tissues, it is synthesized by the tunicates and microorganisms. On an average, around 700 billion tons of cellulose is produced every year. As discussed before for chitosan, cellulose manifests good biocompatibility, biodegradability and low toxicity, and hence is one of the promising substituents for petroleum materials [9-11]. According to cellulose insolubility in most common polar and non-polar solvents, there are limitations in its use as crude materials. Chemically it is considered as a composed of $\beta-1-4$ - linked Dglucopyranosyl units. There is strong inter and intra molecular hydrogen bonds between its chains stabilizing its chemical structure which in turn gives some advantages to cellulose chain molecular structure [12].

In spite of solubility problem some solvents show also the advantage of treatment of cellulose to be able for some kind of chemical functionality. Including for example cuoxam, cadoxen, cuen, ammonium, calcium and sodium thiocyanate, lithium chloride/1, 3-dimethyl-2-imidazolidinone (LiCl/DMI), lithium chloride/N, Ndimethylacetamide $N$-methyl morpholine $N$-oxide (NMMO), ammonia/ammonium salt and phosphoric acid are proven to be effective for solubilizing and molding cellulose to tailored modification [13]. Cellulose as well as its derivatives could produce composites with unique properties and hence applications. Consequently, this generates new composites and/or cellulose derivative, which are termed as ACCs [14]. Starch which is the third member of polysaccharides, it could be chemically described as a structure which with two components namely amylose and amylopectin. It has been reported earlier that, the high-amylose starch could be described as intermediate state which has branched structures connected with branch- 
chains that is longer than amylopectin with molecular weights smaller than that of amylopectin but considered as similar as the structure of amylase [15].

Starch could be also described in other way of description whereas it is used individually or with other member of polysaccharide family specially in food system for many applications not limited to controlling the moisture content as well as water mobility then it could also providing proper texture, these could be further enhances the quality of the product, the stability and finally produce so called cost effective product through controlling and/or even minimizing the production cost [16].

Gelatin is another class of biopolymer but it is belonging to natural proteins. It could be defined as a type of protein which is obtained from the process of denaturation of collagen. Collagen is considered as the main structural protein in different human body systems. As stated for the studied polysaccharides, gelatin is also considered as biocompatible, biodegradable, non-toxic and non-antigenic material [17]. There many reports about improving the mechanical and biological properties by producing it in the form of nanofibers [18-19]. It is stated that, the mechanical properties of nanofibers of gelatin-based materials, is considered among the most important material applied in the field of tissue engineering, it is found to be important but still not very promising according to some limitations and the technique of preparation makes it still of limited properties and still a point of hot topic of research. Hence, the possible applications of biopolymers as individual molecules are nowadays limited because the growing demands in the field of material science require continuous enhancements in materials with special properties. This enhances in a rapid way the blending (composite) of biopolymers with each other in one hand and with other materials in the other hand.

\section{1-2. Biopolymers composites}

\section{1-2-1. Chitosan/Cellulose}

Extensive scientific as well technical efforts have been taken for the development of new materials. Among the most interesting new materials the sustainable composite materials for different applications according to its biodegradability as well as low or even no carbon dioxide release [20]. As indicated earlier chitosan films are 
limited according to insufficient mechanical properties. Functionality could be solving this problem; it may be carried out by reinforcement of nano size cellulose [21]. It is stated that, cellulose is widely used with chitosan to produce composite materials active as antibacterial agents [22], metal ions adsorption [23], odour treatment properties [24], improved water absorption capacity and mechanical characteristics [25], good antistatic and moisture absorption properties [26], high porosity and interconnected porous [27], self-healing characteristics [28], etc. In order to attain comprehensive improvement in electrical, physical, mechanical and thermal characteristics of chitosan-cellulose blend materials tertiary component usually incorporate with it [29]. It could be concluded that, both cellulose and chitosan are promising materials with promising characteristics for composite materials. It is stated from many reports that, biopolymer based materials have relatively poor mechanical, thermal and barrier properties. Adding polymers/nanomaterials are significantly enhancing the properties of the resulted composites. So that, chitosan/nanocellulose composites are now dedicated for many applications according to their new and unique properties.

Such mixing is well known with blending; nowadays it is sophisticated according to the existence of many techniques such as electrospinning, casting and sol-gel method. Despite, extensive research in the field of chitosan based cellulose/nanocellulose composites their commercial applicability is still limited this might be due to their uneconomical production procedure and poor properties as compared to synthetic counter parts. Future studies should be focused on their economical production with synthetic materials like properties [30].

\section{1-2-2. Chitosan/Starch}

Although the chitosan films are poor, the situation in case of starch-based films are different according to excellent oxygen barrier properties, as compared with commercial ethylene vinyl alcohol film [31]. Starch has been used to produce biodegradable films to partially or entirely replace plastic polymers because of its low cost and renewability. However, wide application of starch film is limited by its water solubility and brittleness [32]. 
There are many investigation pointed out that, the starch/ chitosan biofilms are widely applied [33-34]. Since chitosan films are fragile and require plasticizers to reduce the frictional forces between the polymer chains to improve mechanical properties and flexibility, addition of polyols such as glycerol may reduce this drawback.

Most works related to the production of biodegradable films based on starch and chitosan are obtained [35]. In most of these studies, starch is pre-gelatinized prior to chitosan addition and pouring into a mold. Such methods are not adequate to largescale production of films, therefore limiting their industrial application. On the other hand, processing of starch-chitosan by methods such as extrusion and injection molding has been relatively neglected.

\section{1-2-3. Chitosan/Gelatin}

Gelatin exhibits poor mechanical properties [36] and is dissolved quickly in aqueous solutions, which is a drawback for its use as biomaterial. In the last years, several works showed that the mechanical properties and stability of gelatin may be improved using crosslinkers or blending with other polymers [37]. Moreover chitosan and gelatin used together improve the mechanical properties of the scaffolds prepared in comparison with those from single components [38].

Chitosan/gelatin scaffolds were obtained by ice segregation induced self-assembly (ISISA), a versatile, inexpensive technique and environmentally friendly used for the fabrication of porous scaffolds with different sizes, compositions and shapes. ISISA is a cryogenic process based on the unidirectional freezing of a hydrogel, aqueous suspensions or solutions into liquid nitrogen bath $\left(-196{ }^{\circ} \mathrm{C}\right)$ at constant rate and subsequent freeze-drying. Ice crystals grow parallel to the freezing direction and solute particles dispersed in aqueous solutions are exuded to the boundaries between adjacent crystals of ice. After freeze-drying the ice is removed producing porous materials in which a porous network is generated corresponding to the empty areas where ice crystals were initially formed [39-40].

It is stated that, chitosan derivatives are of great concern for many applications. For example, carboxymethyl chitosan, gelatin and nano-hydroxyapatite based injectable gel for bone tissue engineering application [41]. Zhou et al. have demonstrated the 
synthesis and characterization of silver nanoparticles, gelatin and carboxymethyl chitosan hydrogel based antibacterial hydrogels [42]. It is demonstrated that, the influence of carboxymethyl-chitosan and gelatin based hydrogel on cutaneous wound healing [43].

\section{Theoretical and molecular modeling approaches}

Molecular modeling is a theoretical approach whereas chemists are applying the laws of physics to understand the chemical structures. Based upon the laws of physics there are two approaches based on the classical and modern physics. Molecular mechanics based upon the classical physics, electronic structure methods based on quantum mechanics. In the sense of electronic structure methods, the model is an approximation to solve Schrödinger wave equations [44]. This type of modeling could calculate the optimization structure, thermochemical parameters, IR, Raman, NMR as well as many important chemical and physical parameters. Molecular modeling could be useful tool for studying biopolymers as well as their composites.

Regarding the dependence of this computation on Schrödinger equation and the existence of electronic effects this class of computations shows several approximations. In this sense the model could be redefined as an approximation to solve Schrodinger equation. Based upon this consideration electronic structure method has three classes.

1- Semi-empirical method,

2- Ab initio methods,

3- Density functional theory methods.

It is stated that both class of computations are mainly applied to explore materials [45]. Semiemperical quantum mechanical methods as well as ab initio models were utilized together with FTIR spectroscopy for the analyses of both structure and vibrational spectra of glucose and fructose [46]. Molecular modeling was also supporting FTIR to study the interaction between chitosan and Hydroxyapatite [47]. In this work a model is presented for the interaction with electronic properties which is fulfilled later on to improve the model and dedicate this composite as bone replacement [48].Again modeling is supporting spectroscopic tools to investigate the 
electronic properties of some biopolymers and their blends [35] then nano biosensor is designed to act as bio sensor for amino acids.

Both chitosan and cellulose were subjected to extensive investigation to correlate their activity with their structural and vibrational characteristics with different level of theories and FTIR spectroscopy [49]. Extensive molecular modeling work is carried out to estimate the amino acids which constitute gelatin. The modeling was supported with deconvoluated FTIR spectra to understand the structure of gelatin [50]. Chitosan could interact with amino acids, the active sites for interactions also indicated in terms molecular modeling study [51]. Further interaction between chitosan and protein is followed with molecular modeling work [52]. So that, a model for chitosan in nano scale and $\alpha \mathrm{B}$-Crystallin protein is presented. Chitosan dimmer is proved with molecular modeling to act as HIV-1 Protease Inhibitors. The surface is modified with two hydrogen bonding sites to act as inhibitor [53].

Based upon the above considerations, some spectroscopic efforts including recent techniques are needed to manipulate biomaterials. There are many tools of spectroscopy dealing with the detection of biomaterials. Some of them are traditional such as UV-Vis, FTIR, FT-Raman. For better detection and manipulation there are more sophisticated techniques for detection of biomaterials including SERS; FRET; PET, FCS and FCCS.

\section{Surface-Enhancement Raman Scattering Spectroscopy (SERS).}

Raman scattering from a surface of metals is described as enhanced signal as compared to those signals from solutions for example. It is know well known that, metal surface could enhance the Raman signal with $10^{3}-10^{6} \mathrm{X}$ greater than in solution.

This was the idea whereas surface-enhancement Raman scattering is built on, if divalent metals specially silver, but is observable on gold and copper as well. At practical excitation wavelengths, enhancement on other metals is unimportant [5455]. Surface Enhanced Raman Spectroscopy which is termed as SERS technique could be physically defined as a Raman technique that provides greatly enhanced Raman signal from a surface, this surface could be described as Raman-active analyte molecules. This surface could be good substrate for biological or biomaterials to be 
adsorbed onto. Simply for biological system in the form of solution no Raman signal is expected, so that inserting solution of silver in the form of collide could be an active surface in which the biological molecules are adsorbed and then the signal coming from the surface with the details of the biological system on it.

Physically, this process of inserting metal surface-silver- is increasing the intensity of Raman signal on the order of $10^{4}-10^{6}$, and could be in some recent techniques increased as high as $10^{8}$ and $10^{14}$ for some biological and substrate systems [56-57]. The importance of SERS technique is its surface selectivity and highly sensitivity as compared with conventional Raman techniques.

Raman which is ineffective for conventional surface studies because the photons of the incident laser light simply propagate through the bulk and the signal from the bulk overwhelms any Raman signal from the analytes at the surface. By the means of SERS technique with unique silver substrate properties especially in nano scale (in the order of 20-60 nm) selectivity of surface signal results from the presence of surface enhancement (SE) mechanisms only at the surface of silver, gold and also copper. Thus, the surface signal overwhelms the bulk signal, making bulk subtraction unnecessary.

If we are going to describe the mechanisms of enhancement, there are many literatures treating or dealing with is issue. The most common mechanisms for enhancements of the signal could be classified into two main mechanisms, the first one is known as the electromagnetic and the second one is chemical enhancement. While in some cases both mechanisms could be responsible for the enhancement process.

The first one which is the electromagnetic effect is dominant, while the chemical effect is contributing in the process of enhancement by order or two of magnitude [58]. The electromagnetic enhancement which is termed as EME, is depending mainly on the existence of the metal surface with features of so called surface's roughness, on the other hand, chemical enhancement which is termed CE depending on the changes of the adsorbate electronic states according to the process of chemisorption of the analyte [59]. Then, both the structural and the molecular 
identification power of the Raman Signal is used for huge numbers of interfacial systems, including electrochemical, this in turn is very important for biological systems including of course biomaterials specially materials biological molecules and/or macromolecules implemented in the biological systems for certain biological functions, which do very important functions including catalytic, in-situ and ambient analyses and other adsorbate-surface interactions [60-62]. According to the sensitivity of SERS, detection of compounds of low concentrations even at low or trace levels could be accomplished, as well [56].

If someone is going to summarize the process it could be recalled that, the SERS technique is observed primarily for analytes (which is a biological molecule doing certain biological functions) adsorbed onto the surface of divalent metals $\mathrm{Au}, \mathrm{Ag}, \mathrm{Cu}$. Some researchers also tried the phenomena upon some alkali metal surface including $\mathrm{Li}, \mathrm{Na}, \mathrm{K}$, in such cases some changes in laser system took place so that, the excitation wavelength could be near and/or in the visible region [63].

Based upon these findings, one can conclude that, theoretically any metal even divalent or monovalent able of exhibiting Raman Signal Enhancement, but the coinage and alkali metals satisfy calculable requirements and provide the strongest enhancement. Some researchers try to apply another metals such as Pd or Pt they found that these meats exhibit enhancements of about $10^{2}-10^{3}$ for excitation in the near ultraviolet region [57]. It could be also pointed out that, the SERS technique is very important technique with surface selectivity and sensitivity which could be extends Raman Signal utility to a wide variety of interfacial systems which were not previously possible according to absence of surface sensitive agent. This paves the way toward studying systems and molecules including the process in-situ and ambient analysis of electrochemical, catalytic, biological systems and molecules [62-63]. Some researcher's point their research's out toward studying alternative surface techniques, also they try to optimize their work in order to dedicate another enhancement to improve the technique especially for macromolecules and molecules of biological functions with different spectral range to cover different applications with extensive care toward biological molecules [64]. The metal surface can be roughened using one of several methods. The roughness features are on the order of tens of nanometers; small, compared to the wavelength of the incident excitation 
radiation [60-63]. The small size of the particles allows the excitation of the metal particle's surface plasmon to be localized on the particle. The resultant electromagnetic energy density on the particle is the source of the EME mechanism and the primary contributor to the enhancement observed in SERS. As mentioned earlier, Surface Enhanced Raman Scattering (SERS) arises from two mechanisms. The first is an enhanced electromagnetic field produced at the surface of the metal. When the wavelength of the incident light is close to the plasma wavelength of the metal, conduction electrons in the metal surface are excited into an extended surface electronic excited state called a surface plasmon resonance. Molecules adsorbed or in close proximity to the surface experience an exceptionally large electromagnetic field. Vibrational modes normal to the surface are most strongly enhanced. The second mode of enhancement is by the formation of a charge-transfer complex between the surface and analyte molecule.

Many systems of concern are depending on the fact that they are rich in so called electron transfer or electronic transitions according to their nature of charge transfer complexes are in the visible, so that resonance enhancement occurs. They are also characterized by lone pair electrons or pi clouds show the strongest SERS, this was discovered in case of pyridine for the first time. Other aromatic nitrogen or oxygen containing compounds, such as aromatic amines or phenols, are strongly SERS active [65], but can be as high as $600 \mathrm{~nm}$ for larger ellipsoidal silver particles. The plasma wavelength is to the red of $650 \mathrm{~nm}$ for copper and gold [66], the other two metals which show SERS at wavelengths in the 350-1000 nm region. The best morphology for surface plasmon resonance excitation is a small $(<100 \mathrm{~nm})$ particle or an atomically rough surface. SERS can be also used for studying monolayers of materials adsorbed on metals, including electrodes. Many formats could be used rather than electrodes could be used. The most popular include colloids, metal films on dielectric substrates and, arrays of metal particles bound to metal or dielectric colloids through short linkages. Although SERS allows easy observation of Raman spectra from solution concentrations in the micromolar $\left(1 \times 10^{-6}\right)$ range, slow adsorption kinetics and competitive adsorption limit its application in analytical chemistry. 


\section{4- Single molecule spectroscopy (SMS)}

Biological molecules even those described as macromolecules always exist in very low concentrations. Sometimes and/or always they as found as low as single molecule. Investigation of the fate and behavior of these classes of molecules paves the way toward new efforts in the field of spectroscopy which so-called single molecule detection, which is well known branch of spectroscopy of recent applications, called single molecules spectroscopy.

Single molecule spectroscopy (SMS) was born in 1976; the first paper published in single molecule was in April 1976 by T. Hirschfeld [67-68]. From that time the single molecule investigation was oriented only on the soft matter, but the attempts to do single molecule spectroscopy on condensed matter did not stop till it faced great success in 1989 on the hand of the American scientist William Esco Moerner, he continue developing the technique till he awarded Nobel Prize in chemistry at 2014 for his single molecule technique. From its beginning up to the present moment SMS built upon Fluorescence methods, there are some trials to do SMS upon other spectroscopic techniques like single molecule Raman spectroscopy but it is still creeping, so we have to show some facts about fluorescence to understand the coming SMS methods.

The detection limit of SMS went down to fractions of atto Moles (x.x $\times 10^{-24}$ moles) this amount of molecules is equivalent to the reciprocal of Avogadro's No. this means that the single molecule under investigation will be surrounded with billions of buffer molecules in this case one should depend on a good laser source in the excitation to excite only the molecule under study not the host molecules also, this also guarantee a very high signal to noise ratio $(\mathrm{S} / \mathrm{N})$.

Before going on explaining and showing the SM methods, is it necessary to do all these efforts? We can ask the question with other words, why SM spectroscopy is important even critical in some cases in the modern biology, chemistry, and even physics investigations?

The answer is that all the spectroscopic tools before SM methods averaging the total ensemble events, which makes the detection of non-synchronized and heterogeneous events impossible. Moreover when combine single molecule with the high time resolution one could follow the biological time dependent changes and interactions, 
SM spectroscopy became the best tool if nano-structures are the point of investigations.

\section{4-1. Early investigation of SMS}

The early-investigated research in this topic was conducted at low temperature. Through the first SM investigations on (pentacene-doped -p-terphenyl) at $274.5^{\circ} \mathrm{C}$ a shift was recorded in the resonance frequency [69-70] at that time it is called as spectral-diffusion which meant in this situation alterations in the center-frequency of fluorescent molecule. The coupling between the probe and the host molecules influence the frequency of the electronic transition according to the electronic structure changes due to this coupling, this phenomenon has been observed by Moerner and his colleague Ambrose. Now if the illuminating laser kept close to the absorption wavelength, a shift appears as the fluorescent molecule blinks on and off as if it is oscillate between two states one is in resonance with the excitation laser and the other is in out of resonance state. The resulting time traces was explained as the Pterphenyl molecules lies in an interface between the magnetic domains of the lattice cracks. This was an important step in the way of the SM spectroscopy clear the strong of it and sense changes on the nano-scales. During their SM investigation on the polyethylene impure with perylene using the spectral shift method Moerner and Thomas Basché discovered what is called "single molecule hole burning". They have found that by increasing the laser intensity of the spectral shift increased, moreover they have applied the Poisson kinetics statistical model they were able to modify the method to a very powerful tool to do super resolution imaging [71-73].

\section{4-2. Single molecule spectroscopy at room temperature}

Consequence after the studies on the low temperature SM spectroscopy the scientists did not stop trying to reach the room temperature level a brief history is elucidated in the chart in figure 1. The development starts by efforts of Magde, Ellison and others they setup the fluorescence-correlation spectroscopy (FCS) tool [74-75], a lot of work on FCS have been done immediately after they publish their work [76-78]. FCS depends on the passage of the molecule in the laser focal volume. Using advanced electronics and complicated software a co-relation between the burst and the previous one should be done, this co-relation give the opportunity to follow various molecular dynamics. 
The time scales reaches that time to few milli seconds, while it is enhanced to very low time scales also the concentrations enhanced to the level of single molecule level even to sub-single molecule level [79]. The development in the auto-correlation equations helps in this improvement.

SM imaging in room temperature developed very fast and took other dimensions, the work did not depend only on developing the SM systems but the sample preparation also was a point of research, even the sample environment and the glass slide treatment was a point of research. The previous advance leads to the appearance of far-field techniques in the SM field of view, the epi-fluorescence, total internal reflection microscopy (TIRFM) even the conventional wide field microscopy became a powerful tool in SM imaging [80-82] especially in biological, medical, pharmaceutical and biochemical research [83-86].

from the 90's of the last century astonishing collection of SM experiments have been done for single molecules surrounded by various buffers and molecules, during that period SM spectroscopy employed to investigate mainly in biology, bio-physics, biochemistry and cell interactions moreover other studies performed on polymers and in general on molecular dynamics (MD). 


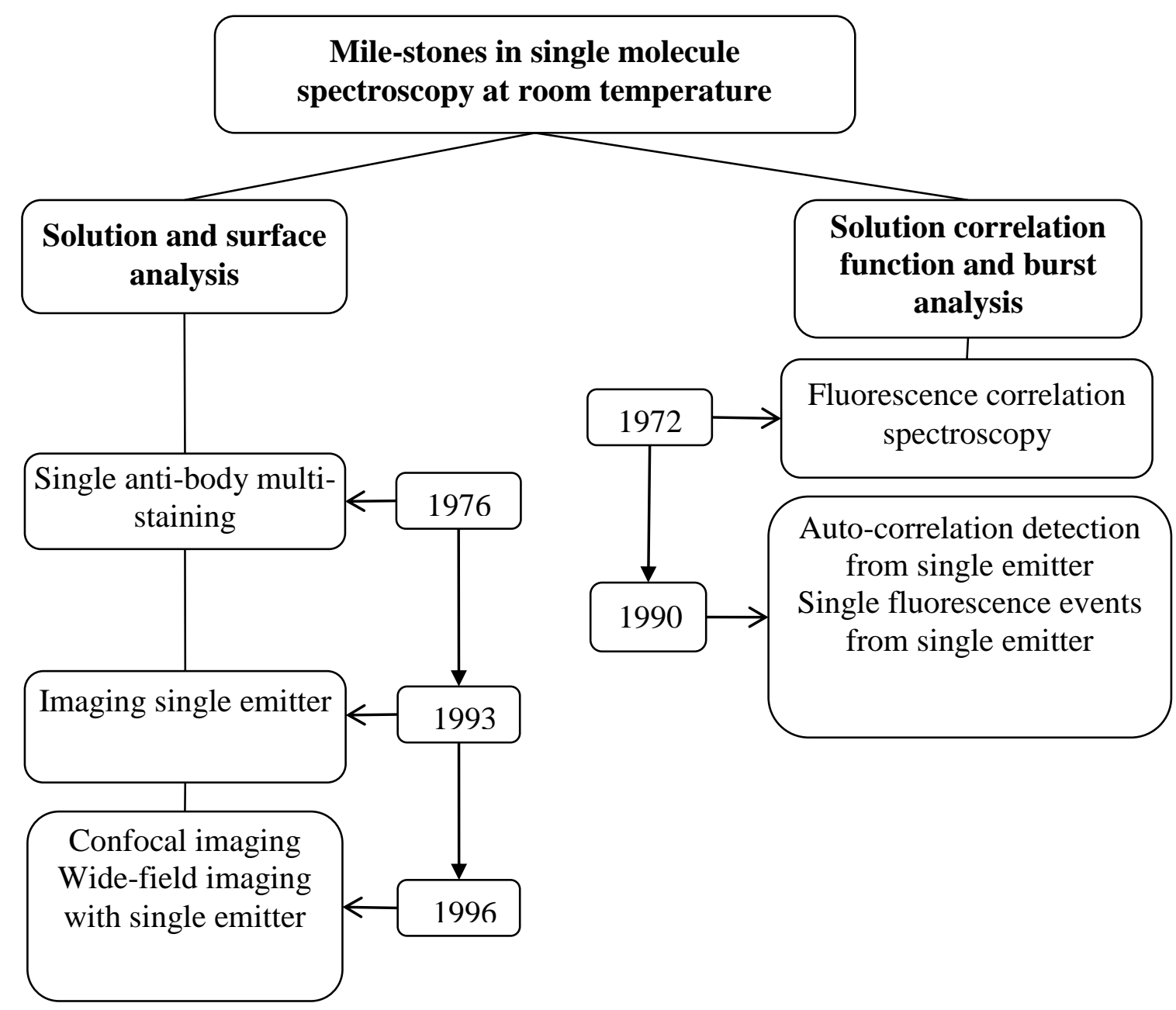

Figure 1. Mile-stones in sing molecule spectroscopy at room temperature.

SM spectroscopy was a powerful tool to change some settled facts like the fact that the protein size increased by the increasing of the concentration of the chemical denaturant, it is discovered during folding unfolding FCS experiments on freely diffused phospho-Glycerat kinase (PGK) enzyme that protein size counteracted and the structure collapsed when the $\mathrm{Gnd} \mathrm{HCl}$ concentration higher than zero and lower than 0.8 mole the same work done on other enzymes like alpha-amylase from Bacillus Licheniformis (BLA), alpha-amylase from Aspergillus oryzae (TAKA) and Porcine pancreatic alpha-amylase (PPA) and the same have been noticed [87-89]. 
The most recent trends to follow up biomaterials are the techniques depending on imaging to get rid of very low concentration and even allow biologist and scientists rather than those working in the field of spectroscopy to deal with biological materials when comparing the images of the studied molecules with standard molecules.

Among the techniques of imaging there well know techniques such as

\section{4-3. Super resolution imaging}

In between the locomotives for SM studies and applications are the bio-molecules investigations alike it was in-vivo, in-vitro or in-situ [83]. The investigation of the biological molecules is often depending on the different varieties of labeling with fluorescent molecules. While fluorescence techniques is non-destructive techniques like the X-ray different methods and electron microscopy, moreover we did not need to crystallize the molecules to single phase crystal which is not easy and in some cases impossible, but the drawback is the spatial resolution. The spatial resolution is limited by what is known as diffraction limitation principle established by Abbe by the end of the nineteenth century [90].

\section{4-4. Fluorescence Life time imaging (FLIM)}

The first paper about FLIM was at 1997 [91] published by B. Herman, life time imaging is a marvelous tool in studying the biological samples especially the cell biology. The outstanding of this technique comes from its added value to the conventional imaging. In the wide field even the confocal imaging the resolution between two successive events is not that good it might be even with the confocal scanning microscope. In this case, the color coding of the lifetime image before overlaying with the intensity image will offer a good resolving between the molecules [92-94]. FLIM is the solution for the auto-fluorescence takes place during in imaging of biological samples in-vivo or in-situ measurements, even inside live cells, that the lifetime is a characteristic for the fluorescent molecule. Regardless its help in resolving the images, the FLIM is a good reporter about the molecule ambient physical micro-parameters, like the micro-viscosity, $\mathrm{PH}$ - value, and the ionic concentration in the buffer, that the fluorophore lifetime is very sensitive to these parameters. FLIM is utilized and upgraded by merging with other methods and techniques like FRET, PLIM and others, details in ref. [95-98]. 


\section{4-5. Single molecule dynamic measurements during molecule diffusion}

Some of the single molecule imaging techniques is presented in the previous sections, the imaging techniques are based on tethered samples, which did not allow freely looking for molecular dynamics and conformation. Hence the solution techniques come strongly to give a helping hand, techniques like fluorescence cross-correlation spectroscopy (FCS), Fluorescence color cross correlation (FCCS), Forester Resonance energy transfer (FRET), Photo-Induced electron transfer (PET) and others.

There also some other techniques dealing with biomaterials as well as other types of biological molecules even throughout but not limited to imaging such as

Fluorescence cross-correlation spectroscopy (FCS)

Forester resonance energy transfer (FRET)

Photo induced electron transfer (PET)

\section{5- Some selective recent applications}

\section{5-1. Organic-inorganic hybrid biomaterials based on functionalized spherosilicates}

Tailor-made spherosilicates and Polyhedral Oligomeric Silsesquioxanes in the form of nano-composites are dedicated as innovative biomaterials [99]. Extensive research work indicated that it could serve as delivery systems, three-dimensional scaffolds for specific tissue engineering, biomaterials for orthopedic, cardiovascular, and reconstructive surgery, etc. The proposed composite in nanoscale is encapsulated as indicated in figure 2 to be ready for medical applications.

\section{5-2. Fabrication of Mg-based implants}

The process of synthesis of $\mathrm{Mg}$ alloys and then possible production of implants from such alloys is carried out with Ball milling in PM encompasses mechanical alloying of two or more alloy powders with hardened steel balls for long periods of time. This process consists of welding, fracturing then rewelding which is resulting in fine microstructure, small grain size and alloying of the powder particles [100].

Table 1 presents the worldwide investigations on porous $\mathrm{Mg}$-based implant through various Pall milling methods with their references. As far the proposed Nanocomposites are implemented in the system a mechanism of interaction is proposed in figure 3. This is what we call the estimated mechanism of $\mathrm{Mg}$ Degradation in vivo. 


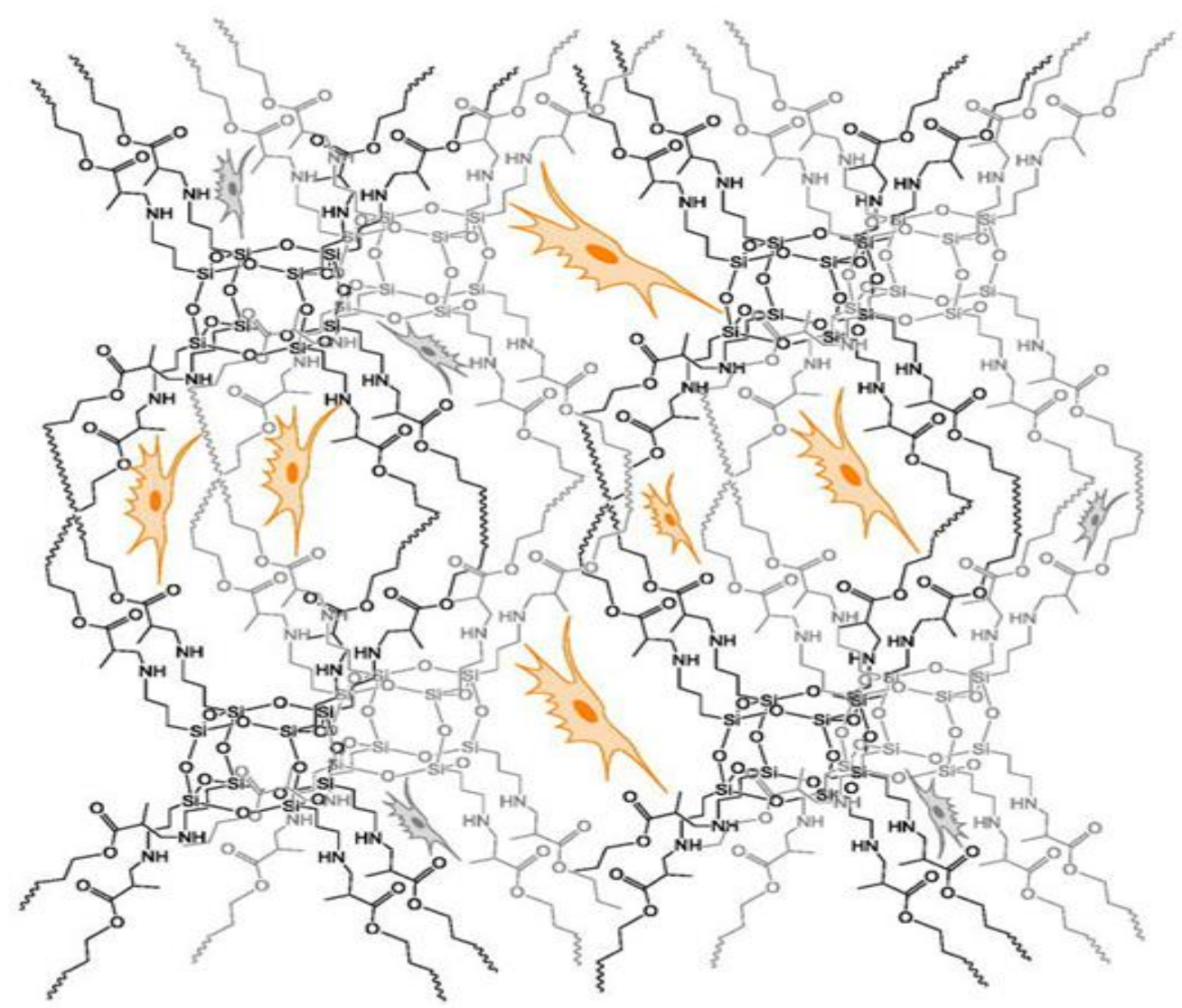

Figure 2. Encapsulated particles in the hybrid network are cells and other structures with functional groups are the proposed nanocomposite.

Table 1. Summary of the studies on the porous Magnesium-based implant using different Pall Milling methods.

\begin{tabular}{|c|c|c|}
\hline Material & Information & Reference \\
\hline $\begin{array}{l}\text { Porous } \quad \mathrm{Mg} / 5 \quad \text { vol\% } \\
\mathrm{Al}_{2} \mathrm{O}_{3}\end{array}$ & $\begin{array}{l}\text { SPS }\left(585^{\circ} \mathrm{C} \text { for } 2 \mathrm{~h} \text { under uniaxial pressing of }\right. \\
20 \mathrm{MPa}) \text {, app. } 60 \% \text { porosity app. } 240 \mu \mathrm{m} \text { grain } \\
\text { size. }\end{array}$ & {$[101]$} \\
\hline $\begin{array}{l}\text { Porous pure } \quad M g \\
\text { scaffolds }\end{array}$ & $\begin{array}{l}0-40 \% \text { porosity, increasing PMMA content } \\
\text { from } 0 \% \text { to } 30 \% \text {, conventional PM (compacted } \\
\text { under } 300 \mathrm{MPa} \text {, heat-treated } 400{ }^{\circ} \mathrm{C} \text { and then } \\
550{ }^{\circ} \mathrm{C} \text { for } 2 \mathrm{~h} \text { ) }\end{array}$ & {$[102]$} \\
\hline $\begin{array}{lrr}\text { Mg } & \text { scaffolds with } \\
\text { increasing } \quad P C L-B G\end{array}$ & $\begin{array}{l}35-40 \% \text { porosity, ammonium hydrogen } \\
\text { carbonate as space holder, conventional PM }\end{array}$ & [103] \\
\hline
\end{tabular}




\begin{tabular}{|l|l|l|}
\hline addition & $\begin{array}{l}(\text { compacted under } 400 \mathrm{MPa} \text { and sintered at } \\
\left.175^{\circ} \mathrm{C} \text { and then } 600{ }^{\circ} \mathrm{C} \text { for } 2 \mathrm{~h}\right)\end{array}$ & \\
\hline Suture anchor screws & $\begin{array}{l}\mathrm{Mg}-0.9 \mathrm{Ca} \text { screw (metallic one) by Metal } \\
\text { injection molding (MIM) }\end{array}$ & {$[104]$} \\
\hline
\end{tabular}



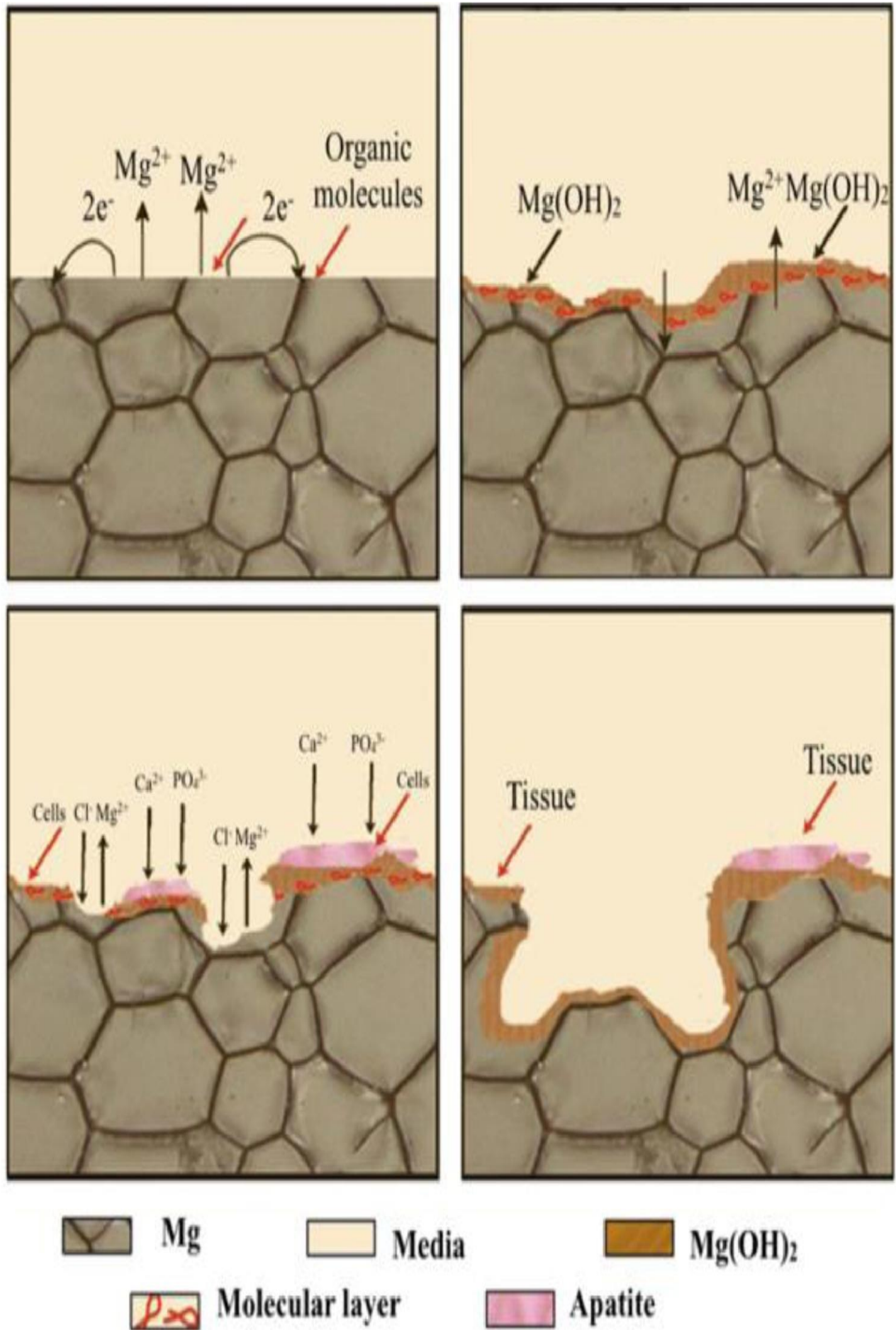

Figure 3. Estimated mechanism of Mg Degradation in vivo [105]. 


\section{5-3. Tissue engineering with conductive biomaterial}

Tissue engineering with biomaterials is not really new route but the most recent trend is the utilization of conductive biomaterials, specially those have biocompatibility which is now widely used to fabricate in vitro platforms for differentiation of progenitor cell population as well as implantable tissue engineering scaffolds. As shown in figure 4 which summarizes the engineering strategies in regulation of different applications including stem cell differentiation, maintenance of phenotypic characteristics, and design of functional implantable scaffolds for better regenerative medicines [106].

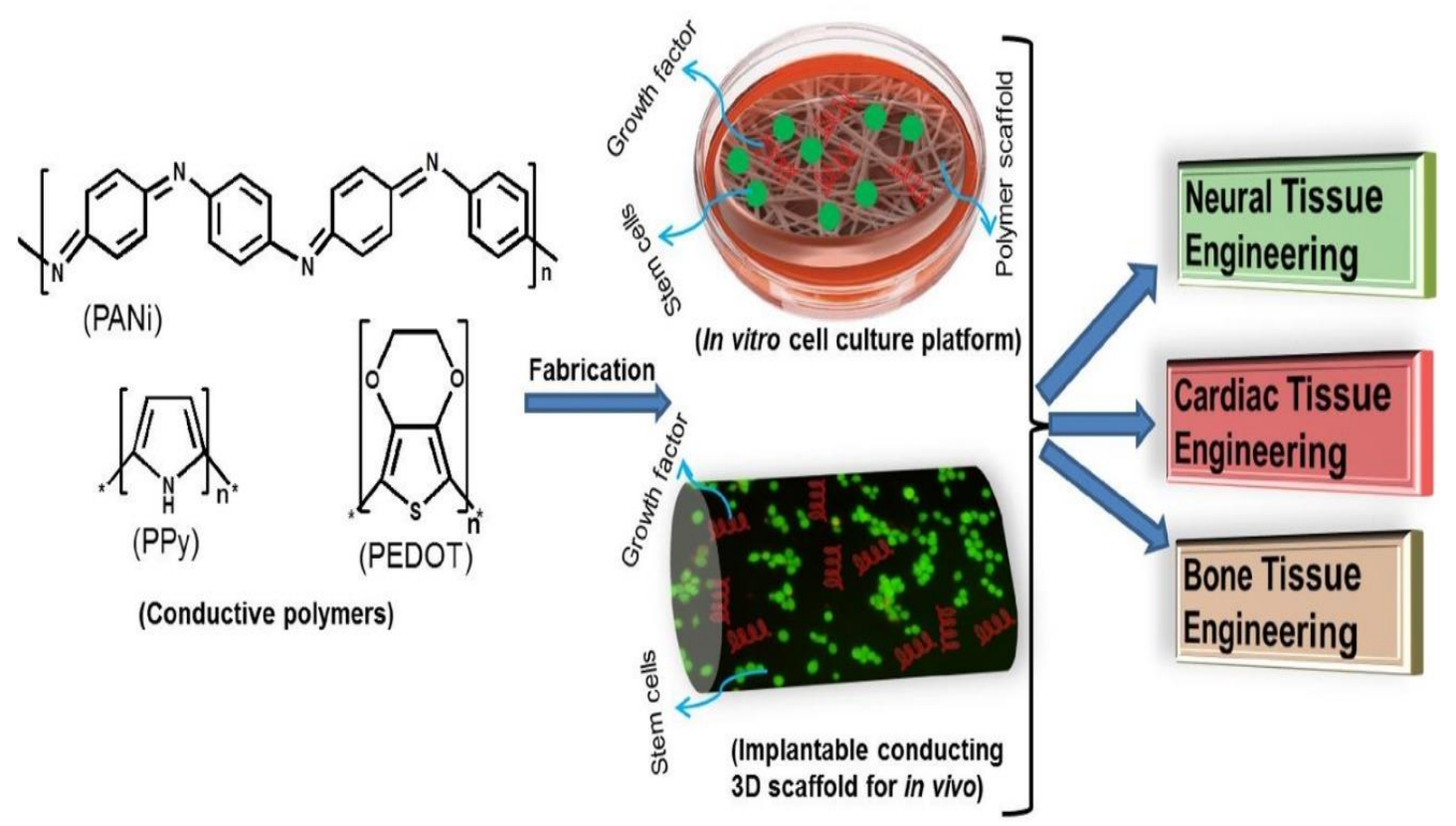


Figure 4. Summary of engineering strategies in regulation of medical applications of conductive biomaterials.

\section{5-4. Biomaterials a fibers for hearts}

Using biomaterials in cardiac heart treatment is very important especially for making new tissue for muscle treatment. Cardiac cell therapy is very important for improving heart function and especially of the permanent failure of muscle functions. Embedding cells into 3D biodegradable scaffolds may better preserve cell survival and enhance cell engraftment after transplantation, consequently improving cardiac cell therapy compared with direct intramyocardial injection of isolated cells [107].

More precise description of such problem could be pointed out as in the following point. The first step is to understand the primary objective of a scaffold used in tissue engineering which is the recreation of the natural $3 \mathrm{D}$ environment most suitable for an adequate tissue growth. Then another important aspect of this commitment is to mimic the fibrillar structure of the extracellular matrix, which provides the essential guidance for cell organization, survival, and function. The overall process is indicated schematically as shown in figure 5; this figure is summarizing schematically and describing of course not in details the technique of preparation.

\section{5-5. Biomaterials for corneal wound healing}

Nowadays it is well know that, the disease which is affecting the cornea of the human eye is the main reason for blindness all over the world [108]. Up to date, the amniotic membrane from pregnant woman after birth which is termed AM is considered the main source used clinically in different hospital for cornea regeneration. Donordependent differences in the AM could result in variable clinical outcomes. This problem could be overcome, and then the proposed biomaterials are currently under investigation for corneal regeneration in vitro and in vivo. Figure 6 highlighted the process as indicated in terms the modern advances in the production of hydrogels, bioengineered prosthetic devices, contact lenses, and drug delivery systems for corneal regeneration. 


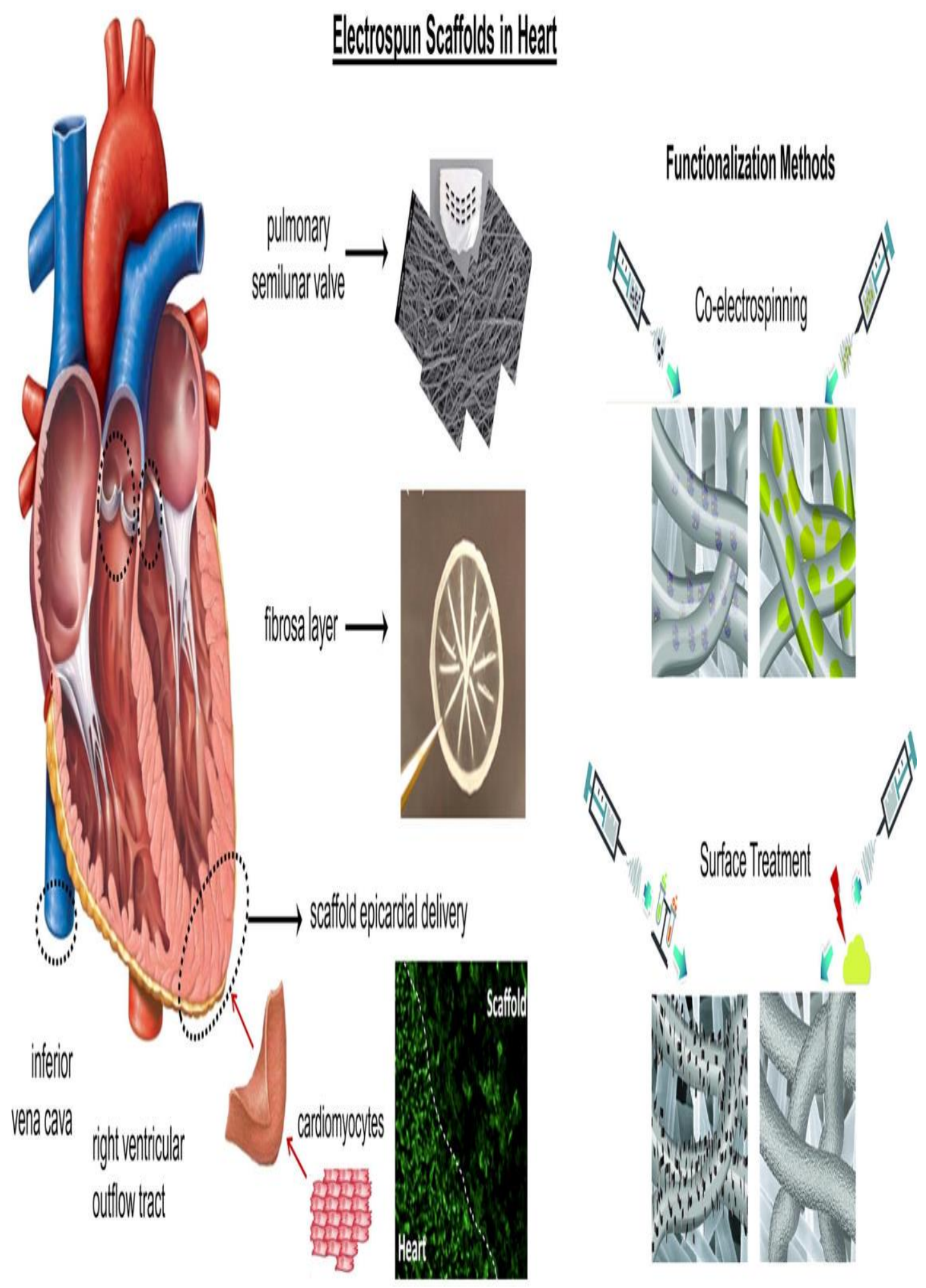

Figure 5. Summary of the process of application of biomaterials as a heart fiber using electrospining technique. 


\section{Hydrogels}

- Collagen

- Gelatin

- Alginate

- Chitosan

- Fibrin

Bioengineered prosthetic devices

- Osteo-odonto keratoprosthesis (O0KP)

- AlphaCor

- Boston keratoprosthesis

\section{Contact lenses}

- Rigid gas-permeable contact lens

- Soft silicone hydrogel contact lens

- Scleral contact lens

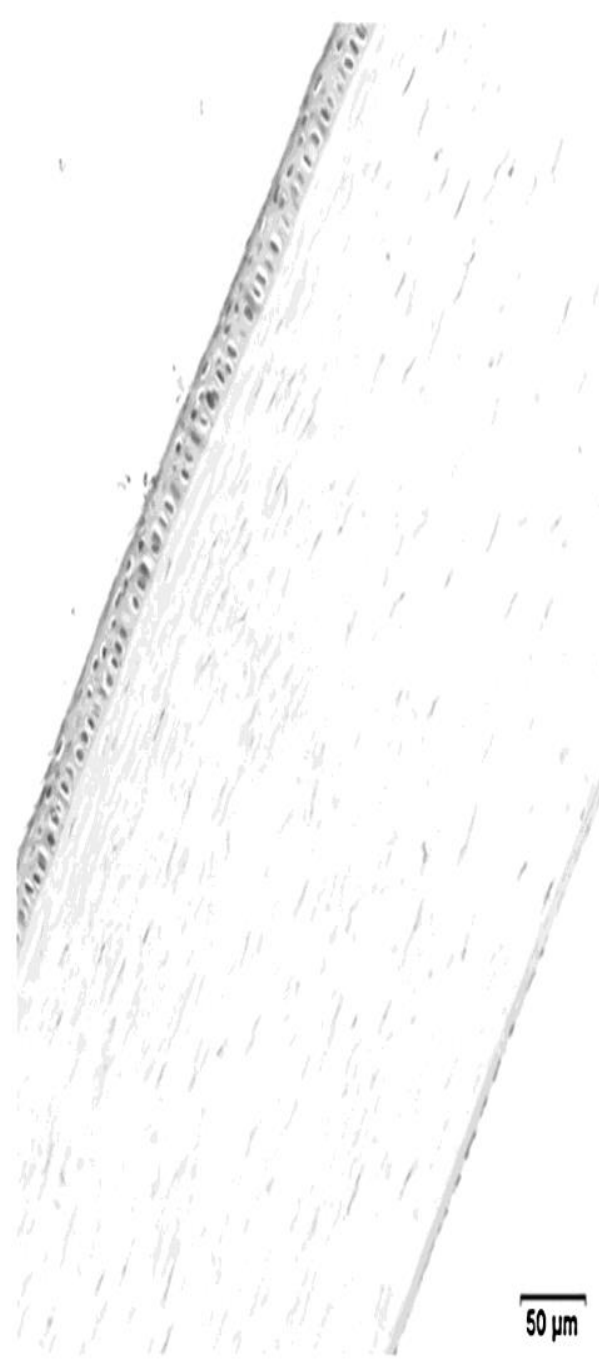

Figure 6. Applications of biomaterials in corneal wound healing.

\section{5-6. Porous dealloying-based biomaterials as a novel biomaterial platform}

The close match of stiffness between implant material and bone is critically important to avoid stress-shielding effect and ensure a fast healing of injured tissues [109]. Here, we introduce liquid metal dealloying method for synthesis of robust open porous biomaterials possessing low Young's modulus. The remarkable advantage of the liquid metal dealloying method is a large flexibility in selecting chemical composition of a desired porous biomaterial together with unique tunable microstructure. Based 
upon the unique combination of mechanical properties of the new open porous $\mathrm{Ti}_{\mathrm{x}} \mathrm{Zr}_{100-\mathrm{x}}$ alloys becomes even more interesting in view of preliminary biological tests highlighting their excellent cytocompatibility. Overall, the liquid metal dealloying provides an opportunity for designing a new biomaterials platform with flexible tunable functionality.
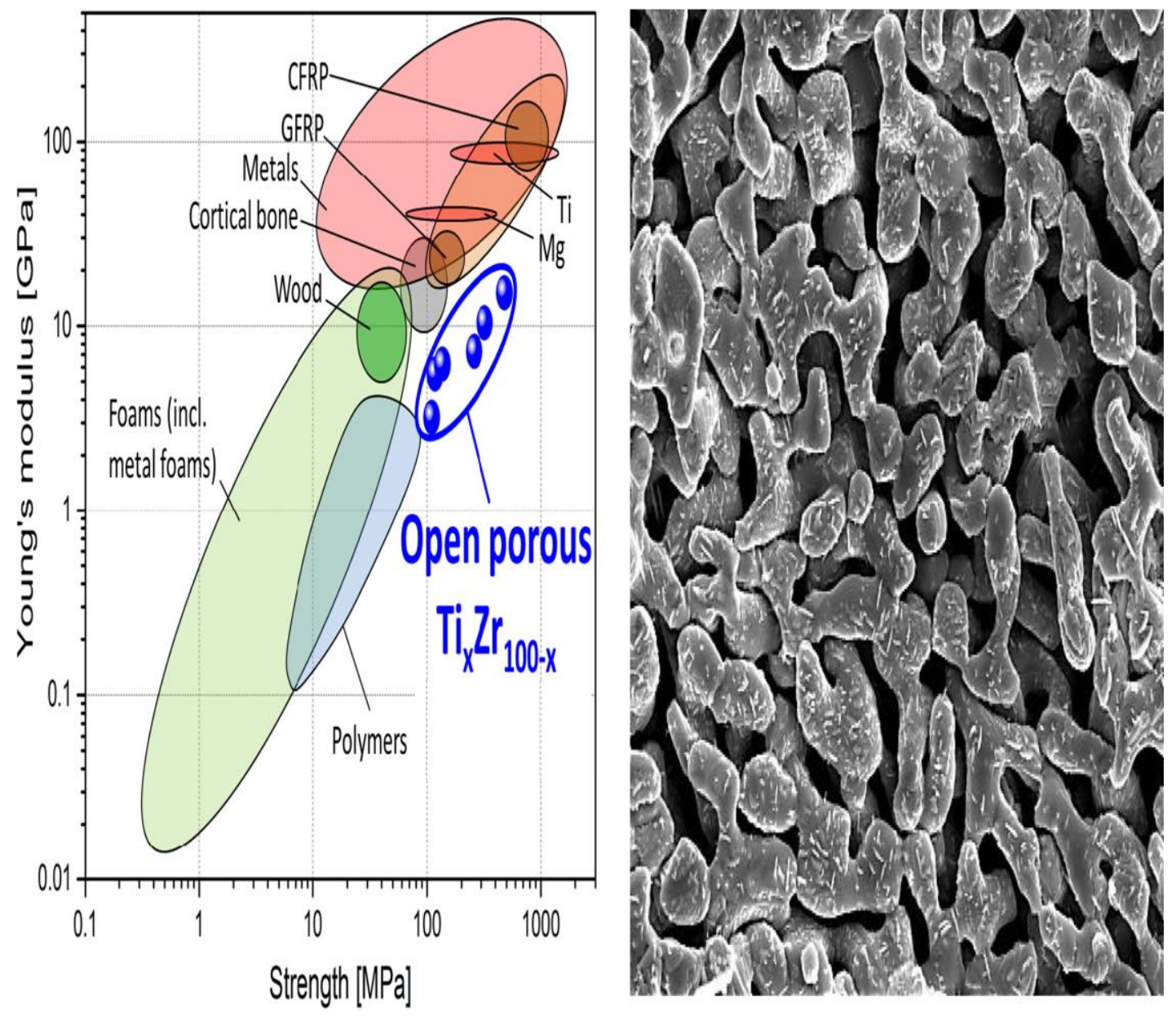

Figure 7. Open porous $\mathrm{Ti}_{\mathrm{x}} \mathrm{Zr}_{100-\mathrm{x}}$ alloys.

\section{6- Conclusion}

Functionality of biomaterials are achieved after blending and/or introduction of matrix include part or full metal oxides nano materials in order to facilitate the process of functionalization based on the desired applications. 
The functionality of biomaterials is providing some advantages which enable it for targeted application these advantages are limited by the available techniques which is a topic of research to continue in the establishment of spectroscopic tools for investigation to follow up the changes in this critical and vital field of research.

Certain care are needed to follow up biomaterials and macromolecules of biological functions according to their sensitive role in one hand and low detection limits on the other hand. Many spectroscopic tools are already introduced including Raman technique, fluorescence as well as techniques based on single molecule detection.

The introduced techniques not only not only depending on conventional spectroscopic methods but also depending on imaging in order to facilitate the fat, transport and the characterization of molecules with biological functions.

Gathering the above statements one can conclude that the field of biomaterial is multidisciplinary field of research and technology it needs an achievements in the basic science to establish model of interactions, it needs innovations in the preparations and characterizations techniques in order to prepare, characterize the materials in proper way for certain application covering the recent and future needs.

\section{7- Acknowledgments}

I dedicate this state of art to the soul of my father, to my family.

I would like also to express my deep thanks to the research group of molecular modeling and molecular spectroscopy at Spectroscopy Department, National Research Centre, for their continuous support during carrying out my research work. 


\section{References}

1- R. Chandra, and R. Rustgi, Prog. Polym. Sci., 23, 1273 (1998)

2- P.K. Dutta, S. Tripathi, G.K. Mehrotra, and J. Dutta, Food Chemistry, 114, 1173 (2009).

3- J. Vinsova, and E.V. avrikova, Curr. Pharmac. Des, 17, 3596 (2011).

4- S. Rivero, M.A. Garcia, and A. Pinotti, Innovative Food Science \& Emerging Technologies, 11,369 (2010).

5- S. Sabnis, and L.H. Block, International Journal of Biological Macromolecules, 27, 181 (2000).

6- D.G. Kim, Y.I. Jeong, C. Choi, S.H. Roh, S. K. Kang, M.K. Jang, et al. Retinol-encapsulated low molecular water-soluble chitosan nanoparticles. International Journal of Pharmaceutics, 319, 130 (2006).

7- M. Rinaudo, Progress in Polymer Science (Oxford), 31, 603(2006).

8- A. Aljawish, L. Muniglia, A. Klouj, J. Jasniewski, J. Scher and S. Desobry, Food Hydrocolloids. 60, 551(2016).

9- K.J. Edgar, C.M. Buchanan, J.S. Debenham, P.A. Rundquist, B.D. Seiler, M.C. Shelton, et al. Advances in cellulose ester performance and application Progress in Polymer Science, 26 (9), 1605(2001).

10- D. Klemm, B. Heublein, H.P. Fink and A. Bohn, Angewandte Chemie-International Edition, 44 (22), 3358(2005).

11- M. Ibrahim, H. Elhaes, D. Atta, JCTN,14,4114(2017).

12- J.A. Figueiredo, M.I. Ismael, C.M.S. Anjo, A.P. Duarte, Cellulose and derivatives from wood and fibers as renewable sources of raw-materials, A.P. Rauter, P. Vogel, Y. Queneau (Eds.), Carbohydrates in sustainable development I: renewable resources for chemistry and biotechnology, Vol. 294, Springer-Verlag Berlin, Berlin, 117(2010).

13- Q. Xu, C. Chen, K. Rosswurm, T. Yao and S. Janaswamy, Carbohydrate Polymers, 149, 274 (2016).

14- Ali Okasha, Diaa Atta, Wael M Badawy, Marina V Frontasyeva, Hanan Elhaes, Medhat Ibrahim.JCTN,14,1357(2017).

15- H. Jiang, M. Campbell, Y. Wu, S. Du, S. Srichuwong and J. L. Jane, Journal of Agricultural and Food Chemistry, 63, 433 (2015).

16- X. Shi, and J.N. BeMiller, Carbohydr. Polym. 50, 7(2002).

17- K. Jalaja, V.S. Sreehari, P.R. Anil Kumar, R. James Nirmala, Mater. Sci. Eng. C 64, 11 (2016).

18- D. Atta, A. Fakhry, M. Ibrahim, Der Pharma Chemica, 7,357(2015).

19- K. Jalaja, D. Naskar, S.C. Kundu and N.R. James, Carbohydr. Polym. 136, 1098 (2016).

20- R. J., Moon, A., Martini, J., Nairn, J., Simonsen, and J. Youngblood, Chem. Soc. Rev., 40, 3941 (2011).

21- S.C. Fernandes, C.S. Freire, A.J. Silvestre, C. PascoalNeto, and A. Gandini. Polymer International, 60, 875 (2011)

22- Y.B. Wu, S.H. Yu, F.L. Mi, C.W. Wu, S.S. Shyu, C. K. Peng, et al. Carbohydrate Polymers, 57, 435 (2004).

23- Z. Liu, H. Wang, C. Liu, Y. Jiang, G. Yu, X. Mu, et al. Chemical Communications, 48, 7350 (2012).

24- D. Atta, F. Gomaa, H. Elhaes, M. Ibrahim, JCTN,14,2405(2017).

25- D. Liu, X. Chen, Y. Yue, M. Chen, and Q. Wu, Carbohydr. Polym, 84, 316 (2011).

26- D. Atta, M.R. Ebrahim, E. AlShkar, Quantum Matter, 5, 207(2016)

27- F. Lv, C. Wang, P. Zhu, and C. Zhang, Cellulose, 21, 4405(2014).

28- J. Duan, C. Han, L. Liu, J. Jiang and J. Li, J. Spectrosc, 5 (2016).

29- W. Xiao, T. Wu, J. Peng, Y. Bai, J. Li, G. Lai, et al. Journal of Applied Polymer Science, $128,1193(2013)$. 
30- H.P. Abdul Khalil, S. Chaturbhuj, K. Saurabh, A.S. Adnan, M.R. Nurul Fazita, M.I. Syakir, Y. Davoudpour, M. Rafatullah, C.K. Abdullah, M.K.M. Haafiz and R. Dungani, Carbohydr. Polym, 150, 216 (2016).

31- P. Forssell, R. Lahtinen, M. Lahelin, and P. Myllärinen, Carbohydr. Polym, 47(2), 125(2002).

32- Q.X. Wu and L.N. Zhang, J. Appl. Polym. Sci., 79, 2006(2001)

33- K.M. Dang and R. Yoksan, Carbohydr. Polym., 115, 575(2014).

34- F.M. Pelissari, F. Yamashita, M.V.E. Grossmann, J. Food Sci. \& Technol. 46 (4) 702 (2011).

35- M. Ibrahim, A. Aziz, O. Osman, A. Refaat, E.M. El-sayed, Spectrochim. Acta, A., 77, 802 (2010).

36- D. Atta, M. Ibrahim, Sensor Letters, 16,467(2018).

37- M. Sarem, F. Moztarzadeh, M. Mozafari, Carbohydrate Polymers, 93 635-643 (2013).

38- H.J. Tseng, T.L. Tsou, H.J. Wang, S.H. Hsu, J. Tiss. Engine. Regen. Med., 7, 20 (2013)

39- S. Deville, E. Saiz, R.K. Nalla, A.P. Tomsia, Science, 311, 515 (2006).

40- C. Suwanchawalit, A.J. Patil, R.K. Kumar, S. Wongnawa, S. Mann, J. Mater. Chem., 19, 8478 (2009).

41- F. Gomaa, A.A. Mahmoud, D. Atta, M. Ibrahim, H. Elhaes, Energy and Environment Focus, 5,330(2016).

42- Y. Zhou, Y. Zhao, L. Wang, L. Xu, M. Zhai, S. Wei, Radiat. Phys. Chem., 81, 553(2012).

43- X. Huang, Y. Zhang, X. Zhang, L. Xu, X. Chen, S. Wei, Mater. Sci. Eng. C, 33, 4816(2013).

44- Y.H. Elshaer, D. Atta, M. A. El-Mansy, M. A. Hegazy, H. A. Ezzat, M. Ibrahim Sensor Letters, 16,311(2018).

45- H. Dorsett and A. White, Overview of Molecular Modelling and Ab initio Molecular Orbital Methods Suitable for Use with Energetic Materials., DSTO Aeronautical and Maritime Research Laboratory, Commonwealth of Australia, AR-011-578 (2000).

46- N.M. Farrage, A.H. Oraby, E.M.M. Abdelrazek, D. Atta, Biointerface Res. Appl. Chem,9, 3934( 2019)

47- O. Osman, A. Mahmoud, D. Atta, A. Okasha, M. Ibrahim, Der Pharma Chemica, 7, 377, (2015).

48- Diaa Atta, Ahmed Refaat, Ahmed Fahmy, Mohamed Ibrahim, Hanan Elhaes, Medhat Ibrahim, Materials Focus, 6,618(2017)

49- M. Ibrahim, A-A. Mahmoud, O. Osman, M. Abd El-Aal and M. Eid, Spectrochim.Acta A. 81, 724 (2011).

50- N.M. Farrage, A.H. Oraby, E.M. Abdelrazek, D. Atta, Egyptian Journal of Chemistry, 62,99(2019).

51- D. Atta, F. Gomaa, H. Elhaes, M. Ibrahim, Energy and Environment Focus, 5,295(2016).

52- Z. Al-Fifi, N.A. Saleh, H. Elhaes and M. Ibrahim, International Journal of Spectroscopy (2015).

53- B. Schrader, 1995, "Infrared and Raman Spectroscopy”, Schrader, B. ed., VCH Publishers Inc.: New York; Chapter 4.

54- A.B. Myers and R.A. Mathies, 1987, "Biological Applications of Raman Spectroscopy: Volume 2: Resonance Raman Spectra of Polyenes and Aromatics”, T. G. Spiro, ed., John Wiley and Sons: New York, Chapter.

55- B. Hecht, B. Sick, U. P. Wild, V. Deckert, R. Zenobi, O. J. F. Martin and D. W. Pohl, 2000, J. Chem. Phys., 112(18), 7761.

56- K. Kneipp, H. Kneipp, I. Itzkan, R. R. Dasar and M. S. Feld, 1999, Chem. Rev., 99, 2957.

57- M. Moskovits, 1985, Rev. Mod. Phys., 57, 783.

58- P. Kambhampati, C. M. Child, M.C. Foster and A. Campion, 1998, J. Chem. Phys., 108, 5013.

59- M.J. Weaver, S. Zou and H. Y. H. Chan, 2000, Anal. Chem., 72, 38A.

60- R.L. Garrell, 1989, Anal. Chem., 61, 401A.

61- S. Zou, C.T. Williams, E. K. Y. and M. J. Weaver, 1998, J. Phys. Chem. B., 102, 9039.

62- G. C. Weaver and K. Norrod, 1998, J. Chem. Ed., 75, 621.

63- A. Campion and P. Kambhampati, 1998, Chem. Soc. Rev., 27, 241.

64- M. Kerker, D.S. Wang, H. Chew, O. Siiman and L.A. Bumm, 1982, "Surface Enhanced Raman Scattering”, R.K. Chang, T.E. Furtak, eds., Plenum Press: New York, 109-128.

65- M. D. Morris, 1992, “Applied Laser Spectroscopy; D. L. Andrews, ed., VCH Publishers Inc.: New York, Chapter 6. 


\section{INTERNATIONAL JOURNAL OF ADVANCED SCIENTIFIC RESEARCH AND INNOVATION}

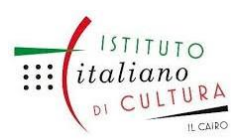

VOLUME 2 , ISSUE $1,2021,25-54$.

www.egyptfuture.org/ojs/

66- T. Hirschfeld, Appl. Opt. 15, 2965-2966, (1976).

67- T. Hirschfeld, Appl. Opt. 15, 3135-3139, (1976).

68- D. Atta, A. Okasha, M. Ibrahim, Der Pharma Chemica, 8,76(2016).

69- J. Schafer, A. Volkmer, C. Subramaniam, G. Striker, C.A.M. Seidel, J. Phys. Chem. A, 103, 331-336, (1999).

70- D. Atta, A. Mahmoud, A. Fakhry, Biointerface Research in Applied Chemistry, 9,3817(2019).

71- M. Sauer, J. Hofkens, J. Enderlein ed. (2011) Handbook of Fluorescence Spectroscopy and Imaging, WILEY-VCH Verlag \& Co. KGaA, Boschstr. 12, 69469 Weinheim, Germany.

72- W. E. Moerner ed. (1988) Topics in Current Physics 44; Persistent Spectral Hole- Burning: Science and Applications, Springer, Berlin, Germany.

73- E.L. Elson, D. Magde, Biopolymers, 13, 1-27, (1974).

74- D.L. Magde, E.L. Elson, W.W. Webb, Biopolymers, 13, 29-61, (1974).

75- R. Rigler, E. Elson ed. (2001) Fluorescence Correlation Spectroscopy, Theory and Applications, Springer, Berlin, Germany.

76- P. Schwille, Cell Biochem. Biophys., 34, 383-408, (2001).

77- S. T. Hess, S. Huang, A. A. Heikal, W. W. Webb, Biochemistry, 41, 697-705, (2002).

78- D. Punj, P. Ghenuche, S. B. Moparthi, J. Torres, V. Grigoriev, H. Rigneault, J. Wenge, WIREs Nanomed.Nanobiotechnol., 6, 268-282,(2014).

79- J.J. Macklin, J.K. Trautman, T.D. Harris, L.E. Brus, Science, 272, 255-258, (1996).

80- T. Funatsu, Y. Harada, M. Tokunaga, K. Saito, T. Yanagida, Nature, 374, 555-559, (1995).

81- D. Atta, A. Okasha, O. Osman, A. Mahmoud, W. Hotaby, Z. Abdel Aziz, A. Fakhry Curr. Sci. Int, 3,199(2014).

82- S. Weiss, Science, 283, 1676-1683 (1999).

83- A. Graslund, R. Rigler, J. Widengren ed. (2009) Nobel Symposium 138 Proceedings, W. E. Moerner, Single Molecule Spectroscopy in Chemistry, Physics and Biology, Springer-Verlag, Berlin, Germany.

84- R. Rigler, M. Orrit, T. Basch'e ed. (2001) Single Molecule Spectroscopy, Nobel Conference Lectures, Springer-Verlag, Berlin.

85- D. Hwan Shin, M. Mamun, J. Almonte, Ch.H. Margraves, Y. Kang, S. Lee, Ch. Choi, Microfluid. Nanofluid., 20, 45, (2016).

86- D. Atta, (2012) Time resolved single molecule fluorescence spectroscopy on surface tethered and freely diffusing proteins. Forschungszentrum-Jülich $\mathrm{GmbH}$.

87- D. Atta, A. Okasha, O. Osman, A. A. Mahmoud, W. El Hotaby, Z. Abdel Aziz, Curr. Sci. Int., 3,199-207,(2014).

88- T. Rosenkranz, R. Schlesinger, M. Gabba, J. Fitter, Chemphyschem, 12, 704-710, (2011).

89- E. Abbe, Arch. Mikrosk. Anat., 9, 413-468, (1873).

90- W. Rettig, B. Strehmel, S. Schrader, H. Seifert eds. (1999) Applied Fluorescence in Chemistry, Biology and Medicine, B. Herman, X. F. Wang, P. Wodnicki, A. Perisamy, N. Mahajan, G. Berry, G. Gordon, Fluorescence Lifetime Imaging Microscopy, Springer-Verlag, Berlin, Germany.

91- E. Hinde, M. A. Digman, K. M. Hahn, E. Gratton, PNAS,110,125-140,(2012).

92- R. Datta, A. Bhattacharjee, A. Hochbaum, E. Gratton, Biophys. J., 112, S1, 186a,(2017).

93- U. Alexiev, P. Volz, A. Boreham, R. Brodwolf, Eur. J. Pharm. Biopharm., 116, 111-124 (2017).

94- E. Gagnon, A. Connolly, J. Dobbins, K. W. Wucherpfennig, Immune. Synapse, 1584, 259-289, (2017).

95- Y. Chen, J. L. Saulnier, G. Yellen, B. L. Sabatini, Front Pharmacol., 7, 46, 2016.

96- S. Kalinina, J. Breymayer, P. Schäfer, E. Calzia, V. Shcheslavskiy, W. Becker, A. Rück, J. Biophotonics, 9, 800-811,(2016).

97- H. Sparks, S. Warren, J. Guedes, N. Yoshida, T. Charn, N. Guerra, T. Tatla, J. Biophotonics,8, 168178, (2015).

98- T. John, Materials Science and Engineering: C, 88, 172-181 (2018)

99- G. Xie, H. Takada, H. Kanetaka, Mater. Sci. Eng. A, 671, 48-53 (2016)

100- M.-H. Kang, T.-S. Jang, S.W. Kim, H.-S. Park, J. Song, H.-E. Kim, K.-H. Jung, H.-D. Jung , Mater. Sci. Eng. C, 62, 634-642 (2016). 
101- Y. Bi, Y. Zheng, Y. Li, Mater. Lett., 161, 583-586 (2015).

102- M. Yazdimamaghani, M. Razavi, D. Vashaee, L. Tayebi, Mater. Sci. Eng. C, 49, 436-444 (2015).

103- M. Wolff, J.G. Schaper, M.R. Suckert, M. Dahms, T. Ebel, R. Willumeit-Römer, T. Klassen, JOM, 68 (4), 1191-1197 (2016).

104- A. Tahmasebifar, Surface Morphology Investigation of a Biodegradable Magnesium Alloy, Middle East Technical University (2015)

105- M. Gajendiran, J. Choi, S-J. Kim, K. Kim, H. Shin, H-J. Koo, K. Kim, Journal of Industrial and Engineering Chemistry, 51, 12-26 (2017)

106- M. Kitsara, O. Agbulut, D. Kontziampasis, Y. Chen, P. Menasché, Acta Biomaterialia, 48, 20-40 (2017).

107- I-L. Tsai, C-C. Hsu, K-H. Hung, C-W. Chang, Y-H. Cheng, Journal of the Chinese Medical Association, 78 (4), 212-217 (2015).

108- I.V. Okulov, A.V. Okulov, I.V. Soldatov, B. Luthringer, R. Willumeit-Römer, T. Wada, H. Kato, J. Weissmüller, J. Markmann, Materials Science and Engineering: C, 88, 95-103, (2018). 